\title{
Integrated approach to the planning of energy consumption by non-traction railway consumers
}

\author{
Alexander Galkin ${ }^{1}$, Alexey Kovalev ${ }^{1, *}$, and Timur Shayuhov ${ }^{1}$ \\ ${ }^{1}$ Ural State University of Railway Transport, Kolmogorova Street, 66, Yekaterinburg, 620034, Russia
}

\begin{abstract}
Non-traction railway load consumes a significant amount of electricity. Russian Railways supplies electricity not only to its structural units but also to other consumers. Private houses, individual entrepreneurs and small production facilities located near the railway are powered by the RZD. It is very important for the company to plan consumption for nontraction needs. The subject of the study in the paper is a systematic approach to the planning of power consumption using the mathematical apparatus of artificial neural networks, correlation analysis and the method of expert assessments. The method of expert assessments allows identifying the most significant factors that affect the consumption of electricity. It is necessary to attract experienced professionals in the field of electricity, working at a particular enterprise. They are able to determine with a high degree of accuracy those factors that have a significant impact on the consumption of the organization. Correlation analysis allows you to mathematically check the degree of influence of a single factor on the resulting value. The apparatus of artificial neural networks allows building a forecast of power consumption, taking into account the influence of external factors. The authors propose to use a systematic approach to the planning of power consumption. It is necessary to combine three tools: the method of expert assessments, correlation analysis and artificial neural networks. The combination of these tools will improve the accuracy of power consumption planning and, as a result, will lead to increased economic efficiency due to the rational consumption of electricity.
\end{abstract}

\section{Introduction}

The consumption of electric energy (EE) is constantly increasing with the development of engineering and technology. In this regard, supplying organizations are forced to set limits and norms on energy consumption, introduce various time-based tariffs to regulate consumption. Consumers are trying to reduce energy costs. Modern enterprises, where the energy management system functions effectively, plan the consumption of electric, thermal and other types of energy, calculate and establish norms, and make energy consumption forecasts. This approach allows implementing a strategy aimed at energy saving and improving energy efficiency.

\footnotetext{
*Corresponding author: akovalev@usurt.ru
} 
Automated control and accounting systems for fuel and energy resources have recently developed significantly. These systems offer great opportunities, such as:

1) collection and processing of information on energy consumption;

2) formation of reports on the activities of the facility on the basis of the automated compilation of various forms and types of information documents;

3) information processing based on scientifically based methodology and decision support;

4) planning of energy consumption of enterprise facilities, monitoring and management of energy consumption.

If the first two possibilities are standard for ASCAPC systems (Automatic system for commercial accounting of power consumption) and their implementation is designed by the manufacturers of these systems, than the remaining possibilities can be implemented using specially developed techniques, models and software.

In the structural subdivisions of JSC "RZD", the planning of the expenditure of energy efficiency on non-traction needs, as a rule, is carried out according to the principle "from the experience of past years".

Such an approach to planning does not take into account the influence of various factors on power consumption, such as air temperature, routine maintenance, commissioning or decommissioning of electrical equipment, modern technical means.

The existing planning system gives a discrepancy between the actual and planned EE expenditure of 5 to $15 \%$, depending on the structural unit. There is also an overestimation of the norms of the future electric power consumption. As a result, unreliable power consumption limits are set, which leads to wasteful spending of money.

In this regard, there is a need to develop a scientifically based model for planning electric power consumption by non-traction consumers.

It should be understood that there are no universal methods of forecasting. For each specific enterprise it is necessary to select its own forecast model. The efficiency of the forecast model depends on a variety of conditions: from the interval and depth of forecasting, time of day, climatic conditions, etc. It is necessary to take into account the peculiarities of the enterprise, possible changes in production processes (increase/decrease in production volume, technical re-equipment), change in ambient temperature, etc. It is important that the forecast model can take into account the factors that lead to a change in the volume of electric power consumption.

For the purposes of forecasting future electric power consumption, the following models are most often used:

1) statistical models of time series: exponential smoothing, regressive and autoregressive ones;

2) structural models of time series: on the basis of classification-regression trees, neural networks, Markov chains.

Analyzing the studies of scientists in the planning of future power consumption, it can be noted that autoregressive (regressive) models, models based on neural networks and exponential smoothing became most widespread. All of them give acceptable forecast accuracy, are relatively easy to use, have the ability to model the influence of external factors.

The complexity of the task of developing a single forecast model that would be suitable for different units that are non-traction consumers is that each of the enterprises has its own specifics and direction of work. Consequently, the factors influencing the power consumption will be different for each structural unit, each individual enterprise.

The mathematical model for planning electric power consumption in railway transport must meet the following requirements:

- be able to identify and take into account a variety of external and internal factors affecting power consumption; 
- be able to set the optimal functional dependence for the process of electric power consumption;

- take into account the random nature of the process under consideration.

In this regard, it is proposed to use an integrated approach to develop such a model. It is necessary to identify the main stages of the development of the model for planning electric power consumption by non-traction consumers.

1. Identification of the main factors affecting the electric power consumption. At this stage, it is necessary to involve specialists who would have an unambiguous understanding of the specifics of the functioning of their structural unit. For example, for production facilities, one of the most important factors will be the number of produced products. And for non-traction consumers of traction substations (TS's own needs), the important factor will be ambient air temperature, technical characteristics of the equipment used at the substation, etc.

2. After identifying the main list of influencing factors, it is necessary to assess the degree of influence of each of them. Factors can be significant and not significant. For these purposes, it is necessary to carry out a correlation analysis between the factors involved and the electric power consumption.

The essence of the correlation analysis lies in the finding of the relations between two random variables. The correlation model based on the experimental data includes the following operations:

1) construction of a correlation plot;

2) compilation of a correlation table;

3) calculation of correlation coefficients;

4) calculation of correlation relations;

5) checking the statistical hypothesis of the significance of the identified relations.

The correlation coefficient gives the most accurate information about the nature and strength of the relation between two factors.

In the process of performing the correlation analysis, it is necessary to check whether the dependence of $y(x)$ is close to linear. To do this, the pair correlation coefficient of the general type is calculated:

$$
p=\frac{\sum_{i=1}^{N} W_{i}\left(x_{i}-\bar{x}\right)\left(y_{i}-\bar{y}\right)}{\sqrt{\sum_{i=1}^{N} W_{i}\left(x_{i}-\bar{x}\right)^{2} \times \sum_{i=1}^{N} W_{i}\left(y_{i}-\bar{y}\right)^{2}}},
$$

where $W_{i}$ - coefficient, which is determined from the initial relations between the variables;

$x, y$-mathematical expectation of the parameters $\mathrm{x}, \mathrm{y}$.

To accelerate the procedure for calculating the correlation coefficients between the values, it is possible to apply data analysis packages, for example, MS Excel and Statistica [3].

In the MS Excel program, the built-in function "CORREL" allows calculating the coefficients of pair correlation.

The Statistica package has a built-in tool "MATRIX OF PAIR CORRELATIONS", which finds pair correlations between the variables (Figure 1). 


\begin{tabular}{|c|c|c|c|c|c|}
\hline \multirow[b]{2}{*}{ Variable } & \multicolumn{5}{|c|}{$\begin{array}{l}\text { Correlation (Book1.sta) } \\
\text { Noted correlations are significant at the level of } \mathrm{p}<, 05000 \\
\mathrm{~N}=12 \text { (Casewise missing data delition) }\end{array}$} \\
\hline & \begin{tabular}{|l|} 
EE consumption, \\
$\mathbf{k W} \mathbf{h}$
\end{tabular} & $\begin{array}{l}\text { Number of produced } \\
\text { products, pcs. (x1) }\end{array}$ & HDD15,5 (x2) & $\begin{array}{l}\text { Losses in the shop } \\
\text { networks, } \mathrm{kW} \mathrm{h}(\mathrm{x} 3)\end{array}$ & $\begin{array}{l}\text { Cost per ton of } \\
\text { aluminum, } \$(x 4)\end{array}$ \\
\hline EE consumption, $\mathrm{kW}$ *h & & 0,72 & 0,48 & 1,00 & 0,36 \\
\hline Number of produced products, pcs. (x1) & 0,72 & & $-0,10$ & 0,72 & 0,79 \\
\hline HDD15,5 (x2) & 0,48 & $-0,10$ & & 0,48 & $-0,46$ \\
\hline Losses in the shop networks, $\mathrm{kW}^{*} \mathrm{~h}(\mathrm{x} 3)$ & 1,00 & 0,72 & 0,48 & & 0,36 \\
\hline Cost per ton of aluminium, $S(x 4)$ & 0,36 & 0,79 & $-0,46$ & 0,36 & \\
\hline
\end{tabular}

Fig. 1. Table of calculation of correlation coefficients obtained in Statistica.

3. As a result of the correlation analysis, the most significant factors are selected. A multiple linear regression equation of the form [2] is compiled:

$$
y=b_{0}+b_{1} x_{1}+b_{2} x_{2}+b_{3} x_{3}+\ldots+b_{n} x_{n},
$$

where: $\mathrm{y}$ - value of electric power consumption;

$\mathrm{x}_{1}-\mathrm{x}_{\mathrm{n}}-$ independent variables (factors that affect electric power consumption);

$\mathrm{b}_{0}-\mathrm{b}_{\mathrm{n}}-$ coefficients of multiple regression.

This equation describes the process of EE consumption.

In this equation, it is required to find the coefficients of multiple linear regression.

The coefficients $b$ in equation (2) can be found in one of three ways:

1) using the Excel function "LINEST". This function calculates statistics for a series using the least squares method;

2) using the "MULTIPLE REGRESSION" tool in the Statistica data analysis package;

3) using the method of least squares:

$$
B=\left[[X]^{T}[X]\right]^{(-1)}[X]^{T} Y
$$

where $\mathrm{B}$ - vector column consisting of the parameters $b_{0}, b_{1}, b_{2}$;

$\mathrm{X}$ - initial matrixx $1, \mathrm{x}_{2}, \mathrm{x}_{\mathrm{n}}$;

$\mathrm{X}^{\mathrm{T}}$ - transposed matrix $\mathrm{x}_{1}, \mathrm{x}_{2}, \mathrm{X}_{\mathrm{n}}$;

$\mathrm{Y}-$ column y.

4. Application of the artificial neural networks (ANN) tool for building the model of electric power consumption planning.

ANN consists of the same type of elements (cells) that simulate the work of brain neurons (Figure 2). Each neuron has its current state similar to the nerve cells of the human brain. These neurons can be excited or hindered. The neuron has a group of synapses unidirectional input connections that are connected to the outputs of the following neurons. In addition, each neuron has an axon. Axon is the output connection of a neuron. The signal goes from it to the synapses of neurons. The synapse is characterized by the magnitude of the synaptic connection or the weight $\mathrm{w}_{\mathrm{i}}$.

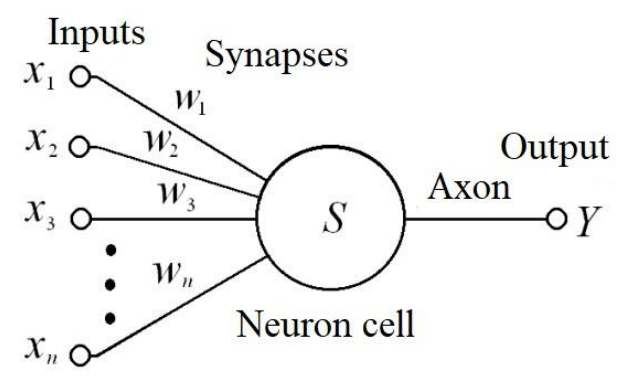

Fig. 2. General view of an artificial neuron. 
The current state of the neuron is calculated as the weighted sum of its inputs:

$$
S=\sum_{i=1}^{n} x_{i} w_{i}
$$

The output of a neuron is defined as a function of its state:

$$
Y=F(S) \text {. }
$$

where $\mathrm{F}$ - nonlinear activation function.

To accelerate the calculation process and provide parallel processing of signals, a large number of neurons are combined into layers. Neurons can be connected both within a single layer and between layers. Figure 3 shows the structure of a multilayer ANN [3].

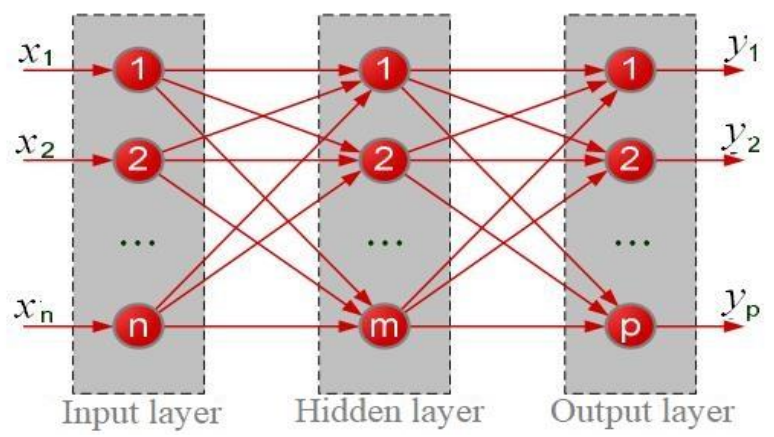

Fig. 3. Structure of an artificial neural network.

Input signals $\mathrm{x}_{\mathrm{i}}$, which are fed to the network, enter the neurons of the input layer. Then they pass through the hidden layers and come out of the neurons of the output layer, thus output signals $y_{i}$ are formed. Signals following the network undergo a series of transformations, which depend on their initial value, as well as on the type of the transforming function and the weight of connection.

An artificial neural network should include the factors identified in the previous stages, taking into account their significance. To train a neural network, there should be data on electric power consumption for previous periods (2-4 steps back) [4].

Among the main advantages of ANN over other forecast models are the following:

- the ability to plan based on a large number of influencing factors;

- high speed of forecasting due to conducting a lot of parallel calculations;

- the ability to detect implicit analogies of precedents of observations;

- the ability to train a neural network.

Thus, the integration of traditional mathematical approaches with a modern apparatus of artificial neural networks makes it possible to increase the efficiency and accuracy of the process of planning electric power consumption. In addition, the potential of automated systems for monitoring and accounting for energy is used more effectively.

\section{References}

1. R.G. Idiyatullin, D.V. Shuralev, A.M. Vdovin, Izvestiia VUZov, Energetika 12 (2001)

2. T.T. Shayuhov, Naukovedenie 9-5 (2017)

3. A.A. Komyakov, A.V. onomarev, O.A. Komyakova, Izvestiia Transsiba, Journal of Transsib Railway Studies 1(5) (2011)

4. A.A. Kovalev, F.S. Nesmelov, A.V. Mikawa, A.A. Kardapolov, N. Isakov, Transport case of Russia 4, 24-26 (2013) 